Objectives: To determine the influence of IRF5 on Immunoglobulin-A vasculitis (IgAV), an inflammatory vascular disease.

Methods: Three IRF5 polymorphisms (rs2004640, rs2070197 and rs10954213) representative of 3 different haplotype blocks were genotyped in 372 Caucasian patients with IgAV and 876 sex and ethnically matched healthy controls.

Results: No statistically significant differences between patients with IgAV and controls were observed when each IRF5 polymorphism was analyzed independently. Similarly, no statistically significant differences between patients with IgAV and controls were found when IRF5 polymorphisms were evaluated combined conforming haplotypes. Additionally, there were no statistically significant differences in genotype, allele and haplotype frequencies of IRF5 when patients with IgAV were stratified according to the age at disease onset or to the presence/absence of gastrointestinal or renal manifestations.

Conclusion: Our results do not support an influence of IRF5 on the pathogenesis of IgAV.

References:

[1] Nat Immunol 2011; 12: 231-8;

[2] Arthritis Res Ther 2014; 16: R146.

Acknowledgments: This study was supported by European Union FEDER funds and "Fondo de Investigaciones Sanitarias" (grant PI18/00042) from 'Instituto de Salud Carlos III' (ISCIII, Health Ministry, Spain). RL-M is a recipient of a Miguel Servet type I programme fellowship from the ISCIII, co-funded by the European Social Fund (ESF, 'Investing in your future') (grant CP16/00033). SR-M is supported by funds of the RETICS Program (RD16/0012/0009) (ISCIII, co-funded by the European Regional Development Fund (ERDF)). VP-C is supported by a pre-doctoral grant from IDIVAL (PREVAL 18/01). LL-G is supported by funds of PI18/00042 (ISCIII, co-funded by ERDF).

Disclosure of Interests: Sara Remuzgo Martinez: None declared, Fernanda Genre: None declared, Verónica Pulito-Cueto: None declared, D. Prieto-Peña: None declared, Belén Atienza-Mateo: None declared, Belén Sevilla: None declared, Javier Llorca: None declared, Norberto Ortego: None declared, Leticia Lera-Gómez: None declared, Maite Leonardo: None declared, Ana Peñalba: None declared, María Jesús Cabero: None declared, Luis Martín-Penagos: None declared, Jose Alberto Miranda-Filloy: None declared, Antonio Navas Parejo: None declared, Javier Sanchez Perez: None declared, Maximiliano Aragües: None declared, Esteban Rubio: None declared, MANUEL LEON LUQUE: None declared, Juan María Blanco-Madrigal: None declared, E. Galindez: None declared, Javier Martin Ibanez: None declared, Santos Castañeda: None declared, Ricardo Blanco Grant/research support from: Abbvie, MSD and Roche, Consultant of: Abbvie, Pfizer, Roche, Bristol-Myers, Janssen and MSD, Speakers bureau: Abbvie, Pfizer, Roche, Bristol-Myers, Janssen, Lilly and MSD, Miguel A González-Gay Grant/research support from: Pfizer, Abbvie, MSD, Speakers bureau: Pfizer, Abbvie, MSD, Raquel López-Mejías: None declared

DOI: 10.1136/annrheumdis-2020-eular.1023

\section{AB0013 HLA ASSOCIATION WITH SYSTEMIC SCLEROSIS (SSC) IN NORTH INDIAN POPULATION AND FAMILIAL INHERITANCE PATTERNS}

S. Machhua ${ }^{1}$, R. Minz ${ }^{2}$, S. K. Sharma ${ }^{3}$, H. Singh ${ }^{2}$, Y. Kumar ${ }^{2}$, S. Anand ${ }^{4}$, S. Handa ${ }^{5}$, S. Singh ${ }^{6} .{ }^{1}$ Post Graduate Institute of Medical Education \& Research, Chandigarh, Immunopathology, Chandigarh, India; ${ }^{1}$ Post Graduate Institute of Medical Education \& Research, Chandigarh, Immunopathology, Chandigarh, India; ${ }^{3}$ Post Graduate Institute of Medical Education \& Research, Chandigarh, Internal Medicine, Chandigarh, India; ${ }^{1}$ Post Graduate Institute of Medical Education \& Research, Chandigarh, Immunopathology, Chandigarh, India; ${ }^{5}$ Post Graduate Institute of Medical Education \& Research, Chandigarh, Dermatology Venereology Leprology, Chandigarh, India; ${ }^{6}$ Post Graduate Institute of Medical Education \& Research, Chandigarh, Department of Pediatrics, Chandigarh, India

Background: It is widely believed that SSc develops in an individual with a permissive genetic makeup.Genetic influences have long been suspected to impact SSc. In families with a history of SSc, the incidence of disease can range from 1.5 to $1.7 \%$ (1). There are several reports of familial occurrence and certain alleles of the HLA system have been associated with the disease (2).No Indian data pertaining to genetic basis of systemic sclerosis is present. Understanding the genetic basis of the disease will help us in defining the biomarkers of the disease in the population that can help in early diagnosis and prognosis.

Objectives: To study HLA association with Systemic sclerosis (SSc) in North Indian Population and its genetic susceptibility to familial systemic sclerosis.

Methods: A total of 150 SSc patients diagnosed by following ACR and EULAR criteria and 150 control subjects, were genotyped for HLA-A, B, DRB1, DQB1 loci by Luminex® 200 Instrument (USA). The association of alleles with disease susceptibility was tested by Chi-square test and Fisher's exact test.
HLA Typing for HLA class I (A, B, C) and II(DR,DQ,DP) for familial study of systemic sclerosis in 2 families was performed by Next Generation Sequencing(NGS) with illumina MiniSeq using MIA FORA NGS Kits from IMMUCOR Antinuclear patterns (ANA) and specific antibodies were detected by indirect Immunofluorescence and Immunoblot (Euroline, Germany).

Results: Strong disease associations were observed for haplotypes $A^{*} 24(\mathrm{OR}=1.7 ;<\quad 0.02), \quad A^{*} 32(\mathrm{OR}=2.8 ;<\quad 0.02), \quad \mathrm{B}^{*} 35(\mathrm{OR}=1.7 ;<\quad 0.03)$ DRB1*11(OR=2.1; $<0.007)$. The reduced frequencies of haplotypes $A^{*} 68(P<$ $0.05), D R B 1^{*} 10(P<0.05), D R B 1 * 12(P<0.00)$ among patients suggested a protective association. There was no statistical association found with HLA $\mathrm{DQB}^{*} 1$.

Through NGS we observed that in the $1^{\text {st }}$ family haplotypes HLA $-A^{*} 11,32,24$; $\mathrm{B}^{*}$ 51, 55, 35; C*-14, 04; DRB1*15, 04; DQB1*05, 03; DPB1*04, 26 appears in affected family members with serological abnormalities. In the $2^{\text {nd }}$ family both mother and daughter had same set of haplotypes except DQB1 with serological abnormalities. The haplotypes DPB $1{ }^{*} 04$ was present in all the diseased individuals of both the families (Fig. 1 and table 1).

Table 1. NGS HLA typing report

\begin{tabular}{lcccccc}
\hline & A & B & C & DRB1 & DQB1 & DPB1 \\
\hline F1 & 1124 & 3515 & 0404 & 1515 & 0505 & 0226 \\
F2 & 1132 & 5155 & 1404 & 1504 & 0503 & 0404 \\
F3 & 1124 & 3555 & 0404 & 1515 & 0505 & 2604 \\
F4 & 3211 & 5115 & 1404 & 1504 & 0503 & 0204 \\
F5 & 2433 & 3544 & 0407 & 1507 & 0502 & 2614 \\
F6 & 1124 & 3555 & 0404 & 1515 & 0505 & 0426 \\
F7 & 1124 & 3555 & 0404 & 1515 & 0505 & 0426 \\
F8 & 2432 & 5135 & 1404 & 0415 & 0305 & 2604 \\
F9 & 1132 & 5155 & 1404 & 1504 & 0503 & 0404 \\
F10 & 1133 & 4452 & 0712 & 1107 & 0203 & 0413 \\
F11 & 1133 & 4452 & 0712 & 1107 & 0303 & 0413 \\
\hline
\end{tabular}

Fig. 1

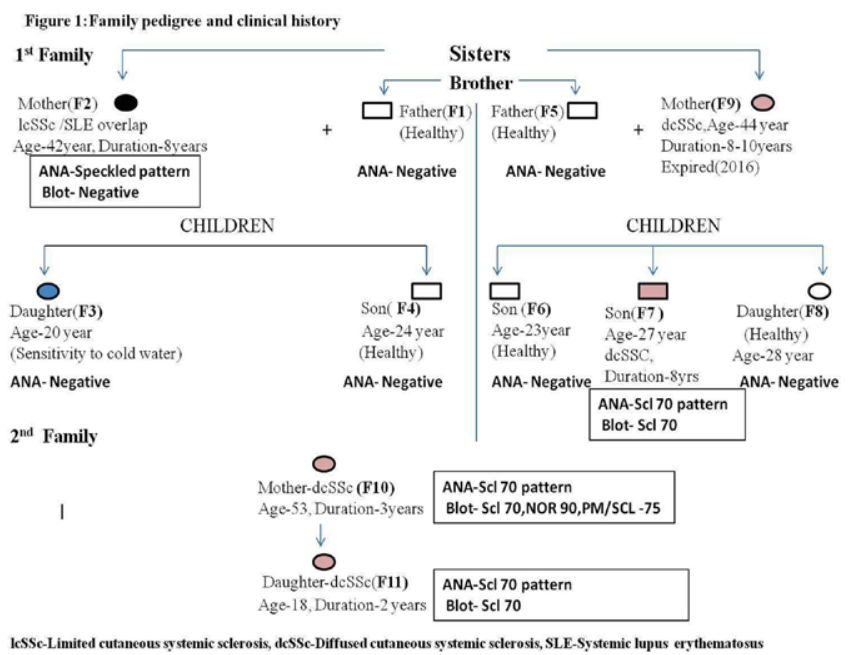

Conclusion: The risk alleles $A^{*} 24,32 ; B^{*} 35$; DRB1 $1^{*} 11$ were found to be associated with North Indian cohort of SSc, while the protecting alleles were $A^{*} 68$; DRB1*10, 12. These risk alleles were present in the SSc affected family members and the protective alleles were absent in the same. Surprisingly, even healthy members carried the same risk alleles but did not manifest the disease or have serological evidence of the same. We have not excluded occurrence of disease at a later age, as presently the healthy siblings are young. Thus our study indicates that though HLA association are found with SSc but many other factors like HLA $\left(H L A{ }^{*} C, D P B 1{ }^{*}\right)$ or non HLA genes as wells as epigenetic factors might also play a role in disease manifestation and severity.

References:

[1] Luo $Y$, Wang $Y$, Wang $Q$, et al. Systemic sclerosis: genetics and epigenetics. J Autoimmun.2013; 41:161-67.

[2] de Juan $\mathrm{MD}^{1}$, Belzunegui J, Belmonte I, Barado J, Figueroa M, Cancio J, Vidal S, Cuadrado E. An immunogenetic study of familial scleroderma. Ann Rheum Dis. 1994 Sep; 53(9):614-7.

Acknowledgments: The technical help of Mr.Manoj Kumar and Mr.Vinkesh are hereby gratefully acknowledged Indian Council of Medical Research(Funding of Fellowship)

Disclosure of Interests: None declared

DOI: 10.1136/annrheumdis-2020-eular.5338 